\title{
Overhead Reduction by Spatial Reusability in Multi-Hop Wireless Networks
}

\author{
Mr. Abhilash Bhise ${ }^{1}$, Dr. Sachin D. Babar ${ }^{2}$ \\ ME Computer Engineering, SIT, Lonavala, Maharashtra, India ${ }^{1}$ \\ Professor, Computer Engg. Dept., SIT, Lonavala, Maharashtra, India ${ }^{2}$
}

\begin{abstract}
The issue of routing in multi-hop wireless networks, to achieve high end-to-end throughput, it is difficult to find the optimal path from the source node to the destination node. Although a large number of routing protocols have been implemented to find the path with minimum transmission time for sending a single packet, such transmission time reduces protocols cannot be guaranteed to achieve high end-to-end throughput. Spatial reusability aware routing in multi hop wireless network is featured by considering spatial reusability of the wireless communication media. Spatial reusability-aware single-path routes and any path routing protocols, and compare them with existing single-path routing and any path routing protocols, respectively. Our evaluation results demonstrate that our protocols significantly improve the end-to-end throughput compared with existing protocols. Resource allocation algorithm is inquired to maximize the energy efficiency (EE) in multiuser decode-and-forward (DF) relay interference networks.
\end{abstract}

Keyword: Routing, wireless network, Hop to Hop to communication.

\section{INTRODUCTION}

Large number of works wireless routing matrices is done in traditional wireless sensor network. In wireless communication network it is important to carefully find the high utility route in multi-hop wireless networks, a large number of routing protocols have been proposed for multi hop wireless networks. However, a fundamental problem with existing wireless routing protocols is that minimizing the overall number of transmissions to deliver a single packet from a source node to a destination node does not necessarily maximize the end-to-end throughput. Single- path routing and any path routing this routing protocols we have to investigate. The task of a single-path routing protocol is to select a cost minimizing path, along which the packets are delivered from the source node to the destination node. In spatial reusability of wireless signals fade during propagation. On the same channel two links are free of interference can transmit at the same time. Most of the existing routing protocols do not take spatial reusability of the wireless communication. We consider spatial reusability of wireless sensor network routing using spatial reusability of by single path routing and any path routing media into account.

This remainder of this paper is organized as follows. Section 2 discusses the related works. Section 3 include system architecture section 4 presents the System analysis. Finally, we conclude the paper and discuss or future work.

\section{LITERATURE SURVEY}

1. A Multi-Radio Unification Protocol for IEEE 802.11Wireless Networks [1].

Algorithm:

- Striping Algorithm

- Round Robin Algorithm

Advantages:

Multiple radios are assigning a flow to a particular channel based on the load across all channels and to maintain this assignment for the duration of the flow.

Disadvantages:

The neighbouring nodes are not able to communicate with each other when that neighbours chosen by orthogonal to the channel and this chooses by wireless node.

2. Highly dynamic destination sequenced distance-vector routing (DSDV) for mobile computers [2].

Algorithm:

- Shortest-path algorithm

- Distributed Bellman-Ford (DBF) algorithm

- dist ante-vector routing algorithm 
Advantage and Disadvantage:

The problems arising with large populations of mobile hosts, which ca $\mathrm{n}$ cause route updates to be received in an order delaying the best metrics until after poorer metric routes are received, we have separated the route tables into two distinct structures.

3. A performance comparison of multi-hop wireless ad hoc network routing protocols [3].

Algorithm:

- Temporally-Ordered Routing

\section{Advantage}

The DSDV over traditional distance vector protocols have advantages that it guarantees loop freedom.

\section{Disadvantage:}

These missing pieces greatly simplify the problem faced by the routing protocol, capture effects, propagation delay, MAC-layer collisions, and the effects of congestion due to large packet sizes are unaccounted for. Furthermore, broadcast and unicast packets were delivered with the same probability, and, as noted in this is not a realistic assumption.

4. Trading structure for randomness in wireless opportunistic routing [4].

Algorithm:

- Computing the number of transmissions each node makes to deliver a packet from source to destination.

- Dijkstra shortest path algorithm.

\section{Advantage}

Field tests on a 20-node wireless tested show that MORE provides both unicast and multicast traffic with significantly higher throughput than both traditional routing and prior work on opportunistic routing.

Disadvantage:

Cant forwarding maximum packet this system.

5. Routing in multi-radio, multi-hop wireless mesh networks [5].

Algorithm:

- Shortest path algorithm.

Advantage and Disadvantage:

First, higher layer software runs unmodified over the ad-hoc network. Second, the ad-hoc routing runs over heterogeneous link layers. Third, while we have currently implemented only the LQSR protocol in the MCL framework, the design, in principle, can support any ad-hoc routing protocol.

\section{SYSTEM ARCHITECTURE / SYSTEM OVERVIEW}

In An ad hoc network wireless sensor nodes dynamically forming a network without the use of any existing network infrastructure administration. Which limit transmission range of wireless network devices, multiple networks "hops" may be needed for one node to exchange data with another across the network. So existing work proposed, at this environment have been developed a variety of new routing protocols targeted specifically, but no realistic performance comparison between them and little performance information on each protocol is available.

1. Importance of spatial reusability

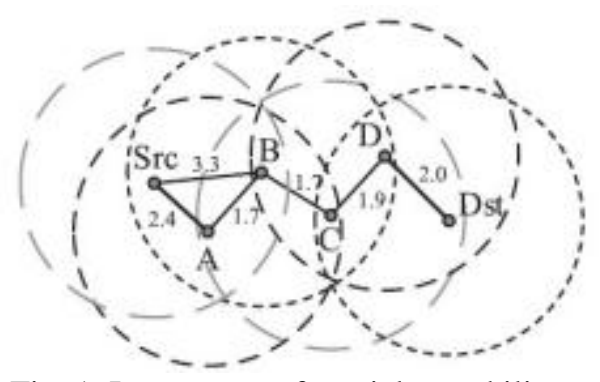

Fig. 1. Importance of spatial reusability 
Here, we use a toy example as shown in Fig. 1 to illustrate the importance of considering spatial reusability of the communication media in single-path routing in wireless networks. In the example, we have four intermediate nodes $\{\mathrm{A}$, B, C, D \} between source node Src and destination node Dst. The dashed circle cantered at each of the nodes indicates the interference range of the node; and the ETX cost is marked beside each of the wireless links.

There are two paths from node Src to node Dst:

Path I: Src-B-C-D-Dst,

Path II:Src-A-B-C-D-Dst.

The ETX cost of path I and path II is $3.3+1.7+1.9+2.0=8.9$ and $2.4+1.7+1.7+1.92 .0=9.7$, respectively. Since path I has a smaller ETX cost, it is normally selected by traditional ETX-based routing protocols, and is expected to have better performance. However, our simulation results show that path II achieves an average end-to-end throughput of $753 \mathrm{Kbps}$, which is 10.2 percent higher than $683 \mathrm{Kbps}$ achieved by path I, when the transmission rate is $11 \mathrm{Mbps}$. This result indicates that the ETX minimizing path is not necessarily the throughput maximizing path in multi-hop wireless networks. If we look into the toy example, we can find that link (Src,A) and link (D, Dst) are out of the interference range of each other, and thus can work simultaneously. Therefore, it is necessary to fuse spatially noninterfering links costs when doing path selection. By fusing costs, we mean that the costs of a set of non-interfering links should be considered as a whole, instead of directly summing them up. In the above example, if we fuse the costs of link (Src,A) and link (D, Dst), and pick the larger cost of the two as the fused cost, the cost of path II becomes 7.7, which is smaller than that of path 2 Thus, when the spatial reusability of wireless communication media is taken into account, the higher throughput path can be selected.

\section{System Model}

In spatial reusability aware routing scheme novel approach is defined with the spectrum spatial reusability in any path routing, and propose SAAR algorithm for participating node selection, cost calculation, and forwarding list determination.

We designed SASR algorithms and SAAR algorithm with different data rates in network simulator. The evaluation results show that our algorithms works improvement to end-to-end throughput compared with existing ones. Specifically, for single-path routing, a throughput gain up to 5.3 with a median of more than 60 percent is achieved in the case of single-flow, and an average gain of more than 20 percent is achieved with multiple flows; for any path routing, a median gain of 13.2 percent and the maximum gain up to 71.6 percent can be realized.

Proposed work presents the results of a detailed packet level simulation comparing four multi-hop wirelesses ad hoc network routing protocols that cover a range.

3. Advantage of Proposed System

- Reduced energy consumption in WSN.

- Secure node to node communication.

- Reduce packet drop attack with trust based active source routing

\section{System Architecture}

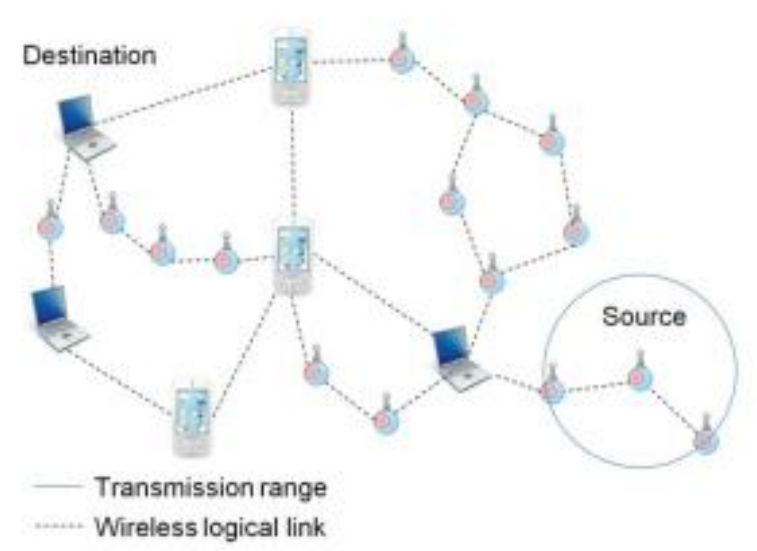

Fig. 2. System Architecture 


\section{Proposed System structure}

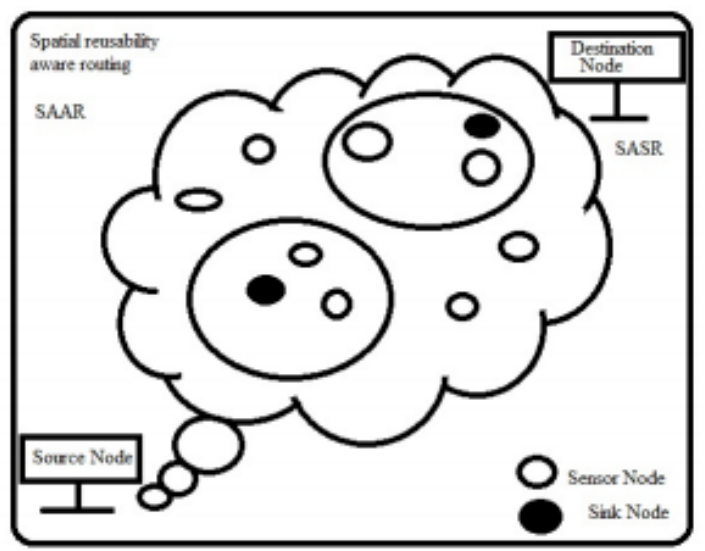

Fig 3. Proposed system structure

\section{Motivation}

Routing protocols are generally implemented based on transmission cost minimizing routing metrics, they cannot guarantee maximum end-to-end throughput when spatial reusability need to be considered. They need centralized control to realize MAC-layer scheduling, and to eliminate transmission contention. The algorithms proposed in this work do not require any scheduling, and the SASR algorithms can be implemented in a distributed manner. Our approach can be extended to adapt to multiple transmission rates, as long as the conflict graph of links can be calculated. Proposed system motivate to simply select the (any) path that minimizes the overall transmission counts or transmission time for delivering a packet. Also in proposed system we manage energy efficient resource allocation for multiuser relay nodes.

\section{Aim}

We aim to exploiting spatial reusability. Specifically, we considered the tradeoff between spatial reuse and data rate, and proposed a decentralized power and rate. Control mechanism work for higher network capacity. We aim to optimum carrier sensing range for better throughput maximization. In Wireless sensor network we implement the quality of a single-hop wireless network. It does not naturally indicate the transmission capability of a source todestination using any path.

8. Goal

1. Reduce Delay: The delay need to reduce in multipath routing because backup routes are identified during route discovery.

2. Load Balancing: The traffic distribution is not equal the network of all links. In some links and bottlenecks when traffic along with multiple routes can spread it can form alleviate congestion in it.

3. Reliability and Fault Tolerance: The original idea behind using multipath routing approach in WSN was to provide path resilience and reliable data transmission. It can cause benefits from the availability of alternative paths to salvage its data packets from node or link failures until a sensor node cannot forward its data packets from node or link failures in the fault tolerance domain.

4. Our goal is to maximize the success ratio: Active routing scheme need to design for maximizing success ratio.

\section{Objectives}

Improve the quality of a single-hop wireless link/hyperlink. It does not naturally show the transmission capability of an end to end path. Minimizing the total number of transmissions to deliver a packet from a source node to a destination node does not necessarily maximize the end-to-end throughput. Proposed efficient energy resource allocation for multiuser relay nodes.

The primary problem is to implement an efficient, robust, scalable routing for reusability of spatial aware routing algorithm. Secure and trustable routing in wireless routing is challenge for spatial reusability of multi hop routing for packet sending data. Network lifetime maximization is bigger challenge in Wireless sensor Network.

\section{Spatial reusability-aware anypath routing}

In contrast to the single-path routing, which restricts the packets to be forwarded through a predetermined path from the source to the destination, anypath routing enables any intermediate node who overhears the packet to participate in packet forwarding. Therefore, in the case of any path routing, our objective is to pick a set of participating nodes $Q$ 
(including the source), and the corresponding profile of distance/cost

$\mathrm{C}=(\mathrm{Ci}) \mathrm{i} \in \mathrm{Q}(1)$ and forwarder lists $\mathrm{F}=(\mathrm{Fi}) \mathrm{i} \in \mathrm{Q}$ to minimize the spatial reusability aware any path cost Csrc. Here, having a smaller $\mathrm{Ci}$ means that node $\mathrm{i}$ is closer to the destination.

Algorithm 1. SASR-MIN Algorithm

This algorithm used for finding minimal path.

$\mathrm{C}=$ Path delivery time

$\mathrm{Q}=$ Covered link

$M^{*}=$ Maximal non-interfering sets

I=non-interfering sets

$\mathrm{P}=$ Path

1. $\mathrm{C} \leftarrow 0$

Initially cost mean delivery time is zero.

2. $\mathrm{Q} \leftarrow \phi$

Covered Set initially empty

3. while $\mathrm{Q} \neq \mathrm{P}$

In this Step loop for $\mathrm{Q}$ is not equal to select path then it executed. Enter in loop.

4. foreach $\mathrm{I} \epsilon M^{*}$ do

In above step for loop executed up to I is an element of set $M^{*}$.

5. I $\leftarrow \mathrm{I} \backslash \mathrm{Q}$

In that Store link in I when set that contains all those link of I that are not in Q.

6. if $\mathrm{I} \neq \phi \quad \& \& \mathrm{c}(\mathrm{I}) /|\mathrm{I}|<$ Threshold

If I is not equals to Empty and also we check value of cost of I up to threshold i.e. particular link.

7. $\propto \leftarrow \mathrm{c}(\mathrm{I}) /|\mathrm{I}|$

Store the value of cost in $\propto$.

8. Temp $\leftarrow$ I

And also non-interfering link are stored in Temp Variable.

In that continuously execute the loop up covered all links are possible .

9. End //End if loop

10. End //End For each loop

11. $\mathrm{C} \leftarrow \mathrm{C}+\mathrm{c}(\mathrm{Temp})$

Store total cost in C.

12. I $\leftarrow \mathrm{I} \cup\{\mathrm{Temp}\}$

Store the non-interfering link in I.

13. $\mathrm{Q} \leftarrow \mathrm{Q} \cup\{\mathrm{Temp}\}$

Stored covered link in $\mathrm{Q}$

14. End // End while loop

15. Return $\mathrm{C}$ and I

First-Fit Algorithm for Min-Cost FusionListing all the maximal non-interfering set on path $\mathrm{P}$ needs $\mathrm{O}\left(3^{\wedge}|\mathrm{P}|^{\wedge} / 3\right)$ time, which is still inefficient when the path $\mathrm{P}$ is long. Therefore, we propose a first-fit algorithm, namely SASR-FF, which can achieve good performance in most of the cases.

Algorithm 2. SASR-FF Algorithm

This algorithm used for minimal cost of path selection from set of paths.

$\mathrm{P}=$ Path

$\mathrm{C}=$ cost of path

$\mathrm{I}=$ non-interfering sets

1. Sort the links in $\mathrm{P}$ by cost in non-increasing order $\mathrm{L}$

2. Initially we give one variable

$\mathrm{K} \leftarrow 0$

3. Cost of path initially zero and I is empty

$\mathrm{C} \leftarrow 0 ; \mathrm{I} \leftarrow \varnothing$

4. Execute the for loop for all links are sorted in $\mathrm{L}$ and $(\mathrm{i}, \mathrm{j})$ are the values are particular node

foreach (i, j) $\epsilon$ L do

5. fused $\leftarrow$ FALSE;

In this step fused is false then go to for loop

6. for $1 \leftarrow 1$ to $\mathrm{k} \mathrm{do}$

In this step variable 1 is equals to one up to $\mathrm{k}$ 
7. reusable $\leftarrow$ TRUE;

If route will be reusable then it assign to TRUE

8. foreach $\left(i^{\prime}, j^{\prime}\right) \in I_{l}$ do

9. if $\left[(i, j),\left(i^{\prime}, j^{\prime}\right)\right] \epsilon$ E then

10. reusable $\leftarrow$ FALSE;

If route is not reusable then assign to FALSE i.e. if node not sending more packet for different route .

11. break;

12. end // end if loop

13. end // end foreach loop

14. if reusable then

If reusable is TRUE

Then find max cost of path add to non-interfering set

15. $I_{l} \leftarrow I_{l} \cup\{(\mathrm{i}, \mathrm{j})\} ; c_{l} \leftarrow \max \left\{\left(i^{\prime}, j^{\prime}\right)\right\}$;

16. fused $\leftarrow$ TRUE;

17. end

18. end

19. if not fused then

20. $\mathrm{k} \leftarrow \mathrm{k}+1$

21. $\quad \mathrm{I} \leftarrow \mathrm{I} \cup\{\{(\mathrm{i}, \mathrm{j})\}\} ; c_{k} \leftarrow t_{i j}$

22. end

23. end

24. for $1 \leftarrow 1$ to $\mathrm{k}$ do

25. $\mathrm{C} \leftarrow \mathrm{C}+c_{l}$

26. end

27. return $\mathrm{C}$ and $\mathrm{I}$

Spatial Reusability - Aware Anypath Routing

We consider hot to exploit spectrum spatial ability for anypath routing in this section. Single path routing restricts the packets to be forwarded through a predetermined path from the source to destination. Intermediate node who overhears the packet to participate in packet forwarding this are enables by any path routing.

Algorithm 3. SAAR Algorithm

Input- $\mathrm{G}=(\mathrm{N}, \mathrm{E})$

A network graph, a source node Src, a destination node Dst.

Output- A set of participating nodes $\mathrm{Q}$, and the corresponding profile of cost $\mathrm{C}$ and forwarder lists $\mathrm{F}$.

1. foreach $i \epsilon \mathrm{N}$ do

i node belong to $\mathrm{N}$

2. $C_{i} \leftarrow+\infty ; F_{i} \leftarrow \emptyset ; \Omega(\mathrm{i}, \mathrm{i}) \leftarrow 1$

Initially $\mathrm{C}$ set infinity, and $\mathrm{F}$ set empty and probability matrix $\Omega$ set 1 for all nodes.

3. end // end foreach loop

4. $C_{D s t} \leftarrow 0 ; \mathrm{q} \leftarrow$ Dst; $\mathrm{Q} \leftarrow\{D s t\} ; \mathrm{I} \leftarrow\{\{$ Dst $\}$;

5. while $\mathrm{q} \neq$ Dst do $\mathrm{q}=$ last picked min-cost node

6. foreach (i, q) $\in E \wedge \mathrm{i} \notin Q$ do

node $\mathrm{I}$ and $\mathrm{q}$ belongs to $\mathrm{E}$ And $\mathrm{i}$ is not belongs to $\mathrm{Q}$

7. $\quad F_{i} \leftarrow F_{i} \cup\{q\} ; \boldsymbol{\Omega}_{i} \leftarrow \Omega$

Add last picked min-cost node into forwarder list $F_{i}$ and probability matrix add node i.

8. foreach $\mathrm{j} \epsilon$ Q do

9. $\boldsymbol{\Omega}_{i}(i, j) \leftarrow 0$

$$
\mathrm{j} \text { is belongs to } \mathrm{Q}
$$

Then set probability matrix set Zero.

10. foreach $\mathrm{k} \in F_{i} \wedge C_{k}<C_{j}$ do

k is belongs to $F_{i}$ and $C_{k}$ is less than $C_{j}$

11. $\boldsymbol{\Omega}_{\boldsymbol{i}}(i, j) \leftarrow \boldsymbol{\Omega}_{\boldsymbol{i}}(i, j)+\omega_{i k} \times \boldsymbol{\Omega}_{\boldsymbol{i}}(k, j)$

Add node link in probability matrix.

12. end //end foreach loop 
13. end //end foreach loop

14. $\left(C_{i}, I_{i}\right) \leftarrow$ CalculateCost $\left(\mathrm{i}, \mathrm{I}, \boldsymbol{\Omega}_{\boldsymbol{i}}, F\right)$;

Calculate minimum cost with node I and interfering set, probability matrix and forwarder list.

15. end //end foreach loop

16. $\mathrm{q} \leftarrow \operatorname{argmin}\left(\mathrm{C}_{\mathrm{i}}\right) ; \mathrm{Q} \leftarrow \mathrm{Q} \cup\{\mathrm{q}\} ; \quad / /$ last picked min-cost node add cost and participating node set $\mathrm{Q}$ into add q. $\mathrm{i} \in \mathrm{N} \backslash \mathrm{Q}$

17. $\Omega \leftarrow \Omega_{\mathrm{q}} ; \mathrm{I} \leftarrow \mathrm{I}_{\mathrm{q}} ;$

Set probability matrix into add last min-cost node and Interfering set also add q.

18. end //end while loop

19. return $Q, C$, and $F$;

Output is participating node and min-cost and forwarding list.

Algorithm 4. Function CalculateCost ()

Input-

$$
\begin{aligned}
& \mathrm{i}=\text { node } \\
& \mathrm{I}=\text { non-interfering sets } \\
& \Omega=\text { a profile of relay probabilities } \\
& \mathrm{F}=\text { a profile of forwarding sets }
\end{aligned}
$$

Output- The fused anypath cost $\mathrm{C}_{\mathrm{i}}$ from node $\mathrm{i}$ to the destination node, and updated non-interfering sets I.

1. fused $\leftarrow$ FALSE; $C_{i} \leftarrow 0$;

Initially fused node is false and Cost set also set zero.

\section{2. foreach $I \in$ I do}

\section{3. reusable $\leftarrow$ TRUE}

If node is reusable then Sst reusable node is true .

\section{4. foreach $\left(j, F_{i}\right) \in I$ do}

Node $\mathrm{j}$ and forwarder node belongs to interfering set

5. if $\left(i, F_{i}\right)$ interfere with $\left(j, F_{i}\right)$ then

6. $\quad$ reusable $\leftarrow$ FALSE; break;

if nodes are not reusable then false.

7. end //end if loop

8. end //end foreach loop

9. if reusable then

10. $\quad \mathrm{I} \leftarrow \mathrm{I} \cup\left\{\left(\mathrm{i}, \mathrm{F}_{\mathbf{i}}\right)\right\}$; fused $\leftarrow$ FALSE ; break;

Add into non-interfering set node $i$ and forwarding list of node $i$. and fused set false.

11. end //end if loop

12. end // end foreach loop

13. if not fused then

14. $I \leftarrow I \cup\left\{\left\{\left(\mathrm{i}, \mathrm{F}_{\mathbf{i}}\right)\right\}\right\}$;

Add into non-interfering set node $i$ and forwarding list of node $i$.

15. end $/ /$ end if loop

16. foreach $I \in I$ do

17. $\mathrm{C}_{\mathrm{i}} \leftarrow \mathrm{C}_{\mathrm{i}}+\mathrm{c}(\mathrm{I})$

Add min-cost with non-interfering set.

18. end// end foreach loop

19. return $C_{i}$ and $I$.

Return cost of set and non-interfering set node.

Algorithm 5. . Euclidean Distance Algorithm

distance $=$ distance

$\mathbf{p}$ and $\mathbf{q}$ are Euclidean Vectors

$\mathrm{f} \mathbf{p}=\left(\mathrm{p}_{1}, \mathrm{p}_{2}, \ldots, \mathrm{p}_{\mathrm{n}}\right)$ and $\mathbf{q}=\left(\mathrm{q}_{1}, \mathrm{q}_{2}, \ldots, \mathrm{q}_{\mathrm{n}}\right)$ are two points in Euclidean n-space, then the distance (d) from $\mathbf{p}$ to $\mathbf{q}$, or from $\mathbf{q}$ to $\mathbf{p}$ is given by the Pythagorean formula:

1. $n=$ number of nodes

2. for $\mathrm{i} \leftarrow 1$ to $\mathrm{n}$ do

3. for $\mathrm{j} \leftarrow 1$ to $\mathrm{n}$ do

4. $\quad$ node $[\mathrm{i}]=[\mathrm{p}, \mathrm{q}]$;

5. node $[\mathrm{j}]=[\mathrm{p}, \mathrm{q}]$; 
6.distance $[\mathrm{i}, \mathrm{j}]=\sqrt{\left((\mathrm{q} 1-\mathrm{p} 1)^{2}\right)+\left((\mathrm{q} 2-\mathrm{p} 2)^{2}\right)+\cdots \ldots \ldots+\left(\left(\mathrm{q}_{\mathrm{n}}-\mathrm{p}_{\mathrm{n}}\right)^{2}\right)}$

$7 . \quad$ end

8. end

9. return distance;

\section{SYSTEM ANALYSIS}

We assess the performance of our SASR and SAAR algorithms in java. Considering that all the nodes use the same transmission rate, we can compare our algorithms with transmission count-based routing protocols and metrics. To be specific, we compared them with the ETX-based DSR [13] (announce by DSR-ETX) and the shortest any path first algorithm [13] based on MORE [12], respectively. Table 1 lists the parameters in our simulation. Specifically, to uniformly distribute 80 nodes in a 2,000 meter to 2,000 meter area, and considered two data rates of 802.11 , including 11 and 54 Mbps. We used CBR to generate 1; 500-byte packets at high enough rates. In addition, RTS/CTS are turned off in all the simulations.

1. Modules:

- Network Creation

- Deciding routing table

- $\quad$ Packet Broadcasting

- $\quad$ Active Source Routing

TABLE I SIMULATION PARAMETERS SETUP

\begin{tabular}{|l|l|}
\hline Parameter & Value \\
\hline Number of Nodes & 80 \\
\hline Terrain Area & $2,000 \mathrm{~m} \times 2,000 \mathrm{~m}$ \\
\hline RTS/CTS & OFF \\
\hline Packet Size & 1,500 Bytes \\
\hline Traffic Generator & CBR \\
\hline CBR Rate & $5 \mathrm{Mbps} / 20 \mathrm{Mbps}$ \\
\hline 802.11 Data Rate & $11 \mathrm{Mbps} / 54 \mathrm{Mbps}$ \\
\hline
\end{tabular}

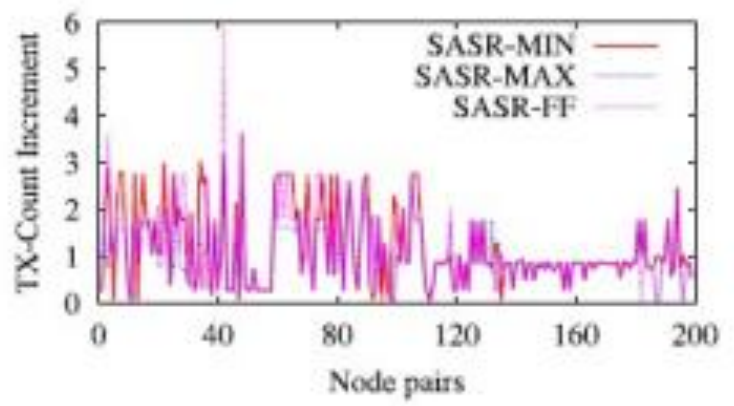

Fig. 4. Overall transmission count increments induced by SASR algorithms
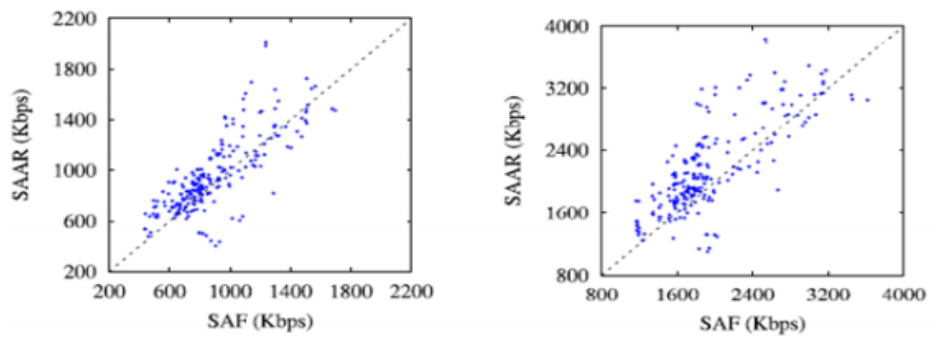

Fig. 5. Pair wise comparisons between SAAR and SAF

\section{CONCLUSION}

Spatial reusability aware routing can efficiently improve the source to destination communication with high end throughput in multi-hop wireless networks, by carefully considering spatial reusability of the wireless communication 
media. This is done by the protocols, SASR and SAAR, for spatial reusability-aware single-path routing and any path routing, respectively. To contribute more for better energy efficiency system implement opportunistic routing to reduce energy consumption. Resource allocation algorithm is inquired to maximize the energy efficiency (EE) in multiuser decode-and forward (DF) relay interference networks.

\section{REFERENCES}

[1] Atul Adya, Paramvir Bahl, Jitendra Padhye, Alec Wolman, Lidong Zhou, "A Multi-Radio Unification Protocol for IEEE 802.11Wireless Networks", in Proc. 1st Int. Conf. Broadband Netw, 2004, pp. 344354.

[2] C. E. Perkins and P. Bhagwat,"Highly dynamic destination sequenced distance-vector routing (DSDV) for mobile computers," in Proc. 4th Annu. ACM/IEEE Int. Conf. Mobile Comput. Netw., 1998, pp. 8597.

[3] J. Broch, D. A. Maltz, D. B. Johnson, Y.-C. Hu, J. G. Jetcheva, "A performance comparison of multi-hop wireless ad hoc network routing protocols", in Proc. 4th Annu. ACM/IEEE Int. Conf. Mobile Comput. Netw. 1998, pp. 8597.

[4] S. Chachulski, M. Jennings, S. Katti, and D. Katabi, "Trading structure for randomness in wireless opportunistic routing”, in Proc. SIGCOMM Conf. Appl., Technol., Archit. Protocols Comput. Commun., 2007, pp. 169180.

[5] R. Draves, J. Padhye, and B. Zill, "Routing in multi-radio, multihop wireless mesh networks", in Proc. 10th Annu. Int. Conf. Mobile Comput. Netw., 2004, pp. 114128.

[6] D. B. Johnson and D. A. Maltz, ”Dynamic source routing in ad hoc wireless networks", in, Mobile Comput, vol. 353,1996 pp. 153181.

[7] T.S. Kim, J. C. Hou, and H. Lim, 'Improving spatial reuse through tuning transmits power, carrier sense threshold, and data rate in multihop wireless networks", in Proc. 12th Annu. Int. Conf. Mobile Comput. Netw., 2006, pp. 366377.

[8] R. P. Laufer, H. Dubois-Ferriere, and L. Kleinrock, "Multirate anypath routing in wireless mesh networks", in Proc. INFOCOM, 2009 , pp. 3745.

[9] Y. Lin, B. Li, and B. Liang, "Codeor Opportunistic routing in wireless mesh networks with segmented network coding", in Proc. IEEE Int. Conf. Netw. Protocols, 2008, pp. 1322.

[10] J. Padhye, S. Agarwal, V. N. Padmanabhan, L. Qiu, A. Rao, and B. Zill, "Estimation of link interference in static multi-hop wireless networks", in Proc. Internet Meas. Conf., 2005, p. 28.

[11] Y. Liu, M. Dong, K. Ota and A. Liu, "ActiveTrust: Secure and Trustable Routing in Wireless Sensor Networks," in IEEE Transactions on Information Forensics and Security, vol. 11, no. 9, Sept. 2016, pp. 2013-2027.

[12] S. Zhao, L. Fu, X. Wang, and Q. Zhang, Fundamental relationship between node density and delay in wireless ad hoc networks with unreliable links, in Proc. 17th Annu. Int. Conf. Mobile Comput. Netw., 2011, pp. 337348.

[13] X. Zhao, J. Guo, C. T. Chou, A. Misra, and S. Jha, A high-throughput routing metric for reliable multicast in multi-rate wireless mesh networks, in Proc. INFOCOM, 2011, pp. 20422050 